\title{
A SURVEY OF CONSISTENCY PROPERTIES IN COOPERATIVE GAME THEORY*
}

\author{
THEO S. H. DRIESSEN†
}

\begin{abstract}
The main purpose of this survey paper is to review the axiomatic characterizations of the Shapley value, the prekernel, the prenucleolus, and the core by means of a consistency property in terms of the reduced games. Whenever possible, new results and new proofs are added.
\end{abstract}

Key words. cooperative game in characteristic function form, reduced game, Shapley value, prekernel, prenucleolus, core, consistency

AMS(MOS) subject classification. 90D12

1. Introduction. Since the introduction of the notion of a cooperative game in characteristic function form in Von Neumann and Morgenstern [30], many solutions for these games have been proposed. In the context of cooperative games in characteristic function form, the solution aims at describing one or more equitable divisions of the total savings among the participants in the game (or, equivalently, fair allocations of the total cost to the users in a joint project). The core is one of the very first proposed "set" solutions and was introduced and named in game theory in Gillies [11], although the idea of the core was already foreshadowed by the Tennessee Valley Authority cost allocation treatment in Ransmeier [22]. Shapley [24] produced an important "one-point" solution, the so-called Shapley value. Moreover, we mention the set solution concept of the prekernel and the one-point solution concept of the prenucleolus.

The mathematical approach to a proposed solution is to examine a number of its (elementary) properties and, if possible, to provide a minimal number of properties which fully characterize the solution. Shapley [24] listed three properties in his characterization of the Shapley value on the class of games with a fixed player set. The main purpose of this paper is to review the characterizations of the Shapley value, the prekernel, the prenucleolus, and the core, on the class of (almost) all games by means of a consistency property. Generally speaking, the formulation of the consistency property for a solution is in terms of the solution itself and the so-called reduced games. This paper reviews the use of reduced games throughout the literature on cooperative games in characteristic function form. Informally, the notion of a reduced game can be elucidated as follows.

A cooperative game is always described by a finite player set as well as a real-valued "characteristic function" on the collection of subsets of the player set. A so-called reduced game is deducible from a given cooperative game by removing one or more players on the understanding that the removed players will be paid according to a specific principle (e.g., a proposed payoff vector). The remaining players form the player set of the reduced game; the characteristic function of which is composed of the original characteristic function, the proposed payoff vector, and/or the solution in question. The consistency property for the solution states that if all the players are supposed to be paid according to a payoff vector in the solution set of the original game, then the players of the reduced game can achieve the corresponding payoff vector in the solution set of the reduced game. In other words, there is no inconsistency in what the players of the reduced game can achieve, in either the original game or the reduced game.

\footnotetext{
* Received by the editors October 24, 1988; accepted for publication (in revised form ) February 20, 1990.

$\dagger$ Department of Applied Mathematics, University of Twente, Enschede, the Netherlands.
} 
We advance three motives for the study of the consistency property in the field of cooperative game theory. First, a distinction between game theoretic solutions can be made by distinguishing between the corresponding consistency properties. Second, the consistency property for a fixed solution may be of importance for the development of the theory of that solution. Third, the consistency property in the game theoretic framework may be useful in order to determine the "consistent" solution of a realistic problem (see $\S 6$ for an application).

In $\S 2$ we bring the framework of cooperative game theory to the forefront and discuss the properties which will be used in the axiomatizations of the solutions as presented in the next sections. In $\S 3$ we review the axiomatic characterization of the Shapley value by means of a consistency property due to Hart and Mas-Colell (Theorem 3.1) and due to Sobolev (Theorem 3.2). Section 4 considers characterization of the prekernel due to Peleg (Theorem 4.1) and of the prenucleolus due to Sobolev (Theorem 4.2), while the axiomatization of the core of balanced and totally balanced games due to Peleg (Theorems 5.1 and 5.2) is reviewed in $\S 5$. In summary, this paper unifies six related theorems that are scattered throughout the literature on mathematics. Whenever new proofs of the existence or uniqueness part of the axiomatizations can be given, they are also included. An additional purpose of this paper is to prove the uniqueness parts of Theorems 3.1, 3.2, and 4.2 by means of a single line of argument. In other cases, we refer to the proofs in the original papers.

2. Properties for solutions of cooperative games. The mathematical model of a cooperative game in characteristic function form is described by a finite nonempty set $N$ and a real-valued function $v$ on the family $2^{N}$ of subsets of the player set $N$, satisfying $v(\varnothing):=0$. We refer to a nonempty subset $S$ of $N$ as a coalition and to $v(S)$ as the worth of coalition $S$. The function $v$ itself is called the characteristic function of the game $(N, v)$. The set of games with player set $N$ is denoted by $G^{N}$, while $G$ denotes the class of all games.

Suppose the grand coalition $N$ is formed, then the players must divide the total earnings $v(N)$ of the grand coalition. A payoff vector for the player set $N$ is a real-valued function $x$ on $N$, denoted by $x \in \mathbb{R}^{N}$. Here $x(i)$, which is usually denoted by $x_{i}$, represents the payoff to player $i \in N$ according to $x \in \mathbb{R}^{N}$. A payoff vector that distributes the amount $v(N)$ among the players is said to be Pareto-optimal (or efficient). A Pareto-optimal payoff vector is also called a pre-imputation and the set of pre-imputations for a game $(N, v)$ is denoted by $I^{*}(N, v)$. Thus,

$$
I^{*}(N, v):=\left\{x \in \mathbb{R}^{N} \mid \sum_{J \in N} x_{J}=v(N)\right\} .
$$

For the sake of convenience, if $x \in \mathbb{R}^{N}$ and $S \subset N$, then we write $x(S)$ instead of $\sum_{j \in S} x_{\jmath}$, where $x(\varnothing):=0$.

A solution is a function $\sigma$ which associates with any game $(N, v)$ a subset $\sigma(N, v)$ of its pre-imputation set $I^{*}(N, v)$. Note that the solution set $\sigma(N, v)$ is allowed to be empty. A solution $\sigma$ is called a value if for any game $(N, v)$ the corresponding solution set $\sigma(N, v)$ is a singleton.

Given any game $(N, v)$, any coalition $T \subset N$, and any payoff vector $x \in \mathbb{R}^{N}$, there are various ways to define a reduced game $\left(T, v^{x}\right)$ with respect to $x$, which is given in terms of the original characteristic function $v$ and the payoff vector $x$. Note that the player set $T$ of the reduced game is obtained here by removing the nonmembers of $T$ from the original player set $N$. The reduced game $\left(T, v^{x}\right)$ should describe the following situation. Suppose that all the players in $N$ agree that the nonmembers of $T$ will be paid 
according to a specific principle (e.g., the payoff vector $x$ ). Moreover, suppose that the nonmembers of $T$ continue to cooperate with the members of $T$. Then the worth $v^{x}(S)$ of coalition $S \subset T$ in the reduced game represents the total savings that the members of $S$ may achieve subject to the foregoing two suppositions.

A solution $\sigma$ is said to possess the reduced game property (RGP) (with respect to a specified type of reduced games) whenever the next condition is satisfied:

RGP. If $(N, v)$ is a game, $\varnothing \neq T \subset N$ and $x \in \sigma(N, v)$, then $x^{T} \in \sigma\left(T, v^{x}\right) . x^{T} \in$ $\mathbb{R}^{T}$ denotes the restriction of $x \in \mathbb{R}^{N}$ to $T \subset N$. Here the reduced game property says that if a payoff vector $x$ is a point in the solution set $\sigma(N, v)$ of the original game $(N, v)$ (according to the solution $\sigma$ ), then the restriction $x^{T}$ of $x$ to any coalition $T \subset N$ belongs to the solution set $\sigma\left(T, v^{x}\right)$ of the corresponding reduced game $\left(T, v^{x}\right)$ (according to the solution $\sigma$ ).

In case the solution is a value, the reduced game condition requires that if the players are supposed to be paid according to the value, then there is no inconsistency in what the players of the reduced game will get, in either the original game or the reduced game. Thus, the RGP can be seen as a property of consistency.

The first version of a reduced game and the corresponding RGP can be found in Davis and Maschler [6]. A systematic study of RGP is presented in Aumann and Drèze [1]. However, their study refers to solutions of games with coalition structures (i.e., partitions of the player set). Usually, solutions of games are defined with reference to the all-player coalition and not with reference to an arbitrary coalition structure. Here we consider the usual treatment of solutions. The core consists of pre-imputations that cannot be improved upon by any coalition. That is, the core of a game $(N, v)$ is defined to be

$$
C(N, v):=\left\{x \in I^{*}(N, v) \mid x(S) \geqq v(S) \text { for all } S \subset N\right\} .
$$

The familiar formula for the Shapley value $\phi(N, v) \in \mathbb{R}^{N}$ of a game $(N, v)$ is as follows (Shapley [24]): for all $i \in N$

$$
\phi_{l}(N, v)=\sum_{S \subset N-\{l\}} \gamma_{N}(S)[v(S \cup\{i\})-v(S)],
$$

where

$$
\gamma_{N}(S):=(|N| !)^{-1}|S| !(|N|-|S|-1) ! \quad \text { for all } S \subset N, \quad S \neq N .
$$

Here $|A|$ denotes the number of elements in the finite set $A$. Note that $\sum_{S \subset N-\{l\}} \gamma_{N}(S)=1$ for all $i \in N$. We also mention an alternative formula for the Shapley value due to Driessen [7], [9]. The Shapley value $\phi(N, v) \in \mathbb{R}^{N}$ of a game $(N, v)$ is equal to

$$
\phi_{i}(N, v)=\sum_{S \subset N-\{i\}} \gamma_{N}(S)[v(N-S)-v(S)] \text { for all } i \in N .
$$

We postpone the definitions of two other major solutions, the prekernel and the prenucleolus. In the remainder of this section we list and discuss several elementary properties for solutions. In the next sections it will be shown that suitably chosen properties together with a consistency property in terms of the reduced games fully characterize a particular solution. We begin by listing the well-known nonemptiness and individual rationality properties for a solution $\sigma$.

NE. A solution $\sigma$ is said to possess the nonemptiness (NE) property if $\sigma(N, v) \neq$ $\varnothing$ for any game $(N, v)$. 
IR. A solution $\sigma$ is said to be individually rational (IR) if for any game $(N, v)$, any $x \in \sigma(N, v)$ and any $i \in N: x_{l} \geqq v(\{i\})$.

RISE. A solution $\sigma$ is said to be relatively invariant under strategic equivalence (RISE) whenever the next condition is satisfied: if $(N, v)$ is a game with $\sigma(N, v) \neq \varnothing$, $\alpha>0$, and $\beta \in \mathbb{R}^{N}$, then $\sigma(N, \alpha v+\beta)=\alpha \sigma(N, v)+\beta$. Here the game $(N, \alpha v+\beta)$ is given by $(\alpha v+\beta)(S):=\alpha v(S)+\sum_{\jmath \in S} \beta$ f for all $S \subset N$.

The relative invariance under $S$-equivalence requires that the solution behave in a natural way with respect to changes in scale that are comparable with positive affine transformations. The following two properties refer to payoffs to "identical" players according to a point in the solution set. Two players are called substitutes if the worth of any coalition containing exactly one of the two players is not affected by interchanging the two players. That is, the players $i, j \in N, i \neq j$, are substitutes in a game $(N, v)$ if $v(S \cup\{i\})=v(S \cup\{j\})$ for all $S \subset N-\{i, j\}$.

SYM. A value $\sigma$ is said to be symmetric (SYM) whenever the next condition is satisfied: if $(N, v)$ is a game, $i \in N, x=\sigma(N, v)$ and $\theta$ a permutation on $N$, then $\theta x=$ $\sigma(N, \theta v)$. Here the game $(N, \theta v)$ and the payoff vector $\theta x \in \mathbb{R}^{N}$ are given by $(\theta v)(\theta S):=$ $v(S)$ for all $S \subset N$ and $(\theta x)(\theta i):=x(i)$ for all $i \in N$.

ETP. A solution $\sigma$ is said to possess the equal treatment property (ETP) if for any substitutes $i, j \in N, i \neq j$, in a game $(N, v)$ and any $x \in \sigma(N, v): x_{i}=x_{\text {j }}$.

The symmetry requires that the value is not affected by a renumbering of the players, while the equal treatment property means that substitutes receive the same payoff by any point in the solution set. It is straightforward to verify that a symmetric value possesses ETP.

Usually, NE, RISE, and ETP must be satisfied to conclude that the solution is standard for two-person games. Here a solution $\sigma$ is called standard for two-person games if for all games $(\{i, j\}, v)$ with $i \neq j$

$$
\sigma_{l}(\{i, j\}, v)=v(\{i\})+\frac{1}{2}[v(\{i, j\})-v(\{i\})-v(\{j\})],
$$

i.e., the "surplus" $v(\{i, j\})-v(\{i\})-v(\{j\})$ is equally divided among the two players according to the solution.

LEMMA 2.1. If a solution $\sigma$ satisfies NE, RISE, and ETP, then $\sigma$ is standard for two-person games.

Proof. Let the solution $\sigma$ satisfy NE, RISE, and ETP and let $(N, v)$ be a two-person game, where $N=\{i, j\}, i \neq j$. Define the two-person game $(N, w)$ by $w(S):=v(S)-$ $\sum_{k \in S} v(\{k\})$ for all $S \subset N$. Because the players $i$ and $j$ are substitutes in the game $(N, w)$, we obtain that $x_{i}=x_{J}=\frac{1}{2} w(\{i, j\})$ for any $x \in \sigma(N, w)=\sigma(N, v)-$ $(v(\{i\}), v(\{j\}))$. As such,

$$
\sigma_{l}(N, v)=v(\{i\})+\sigma_{l}(N, w)=v(\{i\})+\frac{1}{2}[v(\{i, j\})-v(\{i\})-v(\{j\})] .
$$

Since SYM is a stronger requirement than ETP, it follows immediately from Lemma 2.1 that a value is standard for two-person games whenever the value satisfies RISE and SYM. We conclude this section with some notation and definitions. Let $(N, v)$ and $(N, w)$ be two games with the same player set. Then we write $(N, v)=(N, w)$, whenever $v(S)=$ $w(S)$ for all $S \subset N$. Further, for any $S \subset N$ and any $i \in N$, we usually write $S^{c}, S \cup i$, and $S-i$, respectively, instead of $N-S, S \cup\{i\}$, and $S-\{i\}$. Finally, for any $i, j \in$ $N, i \neq j$, we denote the set of coalitions containing player $i$ but not player $j$, by $\Gamma_{l \jmath}$. That is,

$$
\Gamma_{i j}:=\{S \mid S \subset N, i \in S, j \notin S\}
$$


3. On the functional equations determining the Shapley value. Since the introduction of the Shapley value in Shapley [24], several axiomatic characterizations of this value have been given, e.g., in [3], [7], [10], [24], and [31]. The axiomatization of the Shapley value $\phi$ on the class $G^{N}$ of games with a fixed player set $N$ (e.g., in [3]) makes use of four properties: the Pareto-optimality, the so-called dummy player property, the symmetry (the equal treatment property, respectively), and the additivity property, i.e.,

$$
\phi(N, v+w)=\phi(N, v)+\phi(N, w) \text { for all games }(N, v) \text { and }(N, w) .
$$

Here the game $(N, v+w)$ is given by $(v+w)(S):=v(S)+w(S)$ for all $S \subset N$.

We recall that the additivity axiom is the most essential one in the above characterization of the Shapley value on $G^{N}$ since the first three axioms are required to be satisfied in order to determine the value on the so-called unanimity games which form an additive basis for the linear space $G^{N}$ ( see [24]). However, the additivity axiom is no longer useful for characterizing the Shapley value on the class $G$ of all games.

First, we pay attention to the axiomatization of the Shapley value on $G$ with the aid of a consistency property in terms of the reduced games as defined in Hart and MasColell [13]. They consider a type of reduced games dealing with the so-called subgames of a fixed game. Given any game $(N, v)$ and any coalition $T \subset N$, the $\operatorname{subgame}(T, v)$ of $(N, v)$ on $T$ is obtained by restricting the characteristic function $v$ to subsets of $T$ only, i.e., to $2^{T}$.

Given any value $\sigma$ on $G$, any game $(N, v)$ with $|N| \geqq 2$ and any player $i \in N$, the corresponding reduced game $\left(N-\{i\}, v^{\sigma}\right)$ with respect to the value $\sigma$ is as follows: for all $S \subset N-\{i\}$

$$
v^{\sigma}(S):=v(S \cup\{i\})-\sigma_{i}(S \cup\{i\}, v) .
$$

Note that the player set of the reduced game is obtained here by removing only one player of the original player set. Furthermore, the worth of any coalition in the above reduced game is equal to the original worth of the coalition together with the single player minus the payoff to the single player, according to the value in the subgame with the coalition together with the single player as player set. The reduced game property requires that the value yields the same payoffs to the players in the reduced game in comparison with the original game, i.e.,

$$
\sigma_{J}\left(N-\{i\}, v^{\sigma}\right)=\sigma_{J}(N, v) \text { for all } j \in N-\{i\} .
$$

THEOREM 3.1 (Hart and Mas-Colell [13]). The Shapley value $\phi$ on $G$ is the unique value on $G$ which possesses RISE, ETP, and RGP with respect to the reduced game of $(3.1)$.

Second, we mention another type of reduced games considered by Sobolev [27] in order to axiomatize the Shapley value on $G$.

Given any game $(N, v)$ with $|N| \geqq 2$, any player $i \in N$, and any payoff vector $x \in$ $\mathbb{R}^{N}$, the corresponding reduced game $\left(N-\{i\}, v^{x}\right)$ with respect to $x$ is as follows: for all $S \subset N-\{i\}$

$$
v^{x}(S):=(|N|-1)^{-1}|S|\left[v(S \cup\{i\})-x_{l}\right]+(|N|-1)^{-1}(|N|-|S|-1) v(S) .
$$

Note that the worth of any coalition in the above reduced game is obtained as a convex combination of the worth of the coalition in the original game and the original worth of the coalition together with the single player minus the payoff $x_{l}$ to the single player $i$ for his participation.

THEOREM 3.2 (Sobolev [27]). The Shapley value $\phi$ on $G$ is the unique value on $G$ which possesses RISE, SYM, and RGP with respect to the reduced game of (3.2). 
Next, we list a third type of reduced games in order to state a third and last consistency property for the Shapley value. This type of reduced games is derived from the alternative formula (2.3) for the Shapley value.

Given any game $(N, v)$ with $|N| \geqq 2$, any player $i \in N$, and any payoff vector $x \in$ $\mathbb{R}^{N}$, the corresponding reduced game $\left(N-\{i\}, v^{x}\right)$ with respect to $x$ is as follows:

$$
\begin{aligned}
& v^{x}(\varnothing):=0, \quad v^{x}(N-\{i\}):=v(N)-x_{i}, \quad \text { for all other } S \subset N-\{i\}, \\
& v^{x}(S):=(|N|-1)^{-1}(|N|-|S|-1)[v(S)-v(N-S)]-(|N|-1)^{-1}|S| x_{l} .
\end{aligned}
$$

THEOREM 3.3 (Driessen). The Shapley value $\phi$ on $G$ possesses the RGP with respect to the reduced game of (3.3).

We conclude this section by proving the existence part of Theorem 3.2 as well as the uniqueness part of Theorems 3.1, 3.2. For a proof of the existence part of Theorem 3.1 (i.e., the proof of the RGP of the Shapley value with respect to the reduced game of (3.1)), we refer to Hart and Mas-Colell [13]. Our purpose is to prove the uniqueness parts of Theorems 3.1, 3.2 (as well as the forthcoming Theorem 4.2) by means of one specified argumentation. In other words, the uniqueness proofs are highlighted by placing the proofs in the context of a single line of argument.

Proof of the existence part of Theorem 3.2. It is well known that the Shapley value $\phi$ on $G$ possesses SYM and RISE. Thus, it remains to prove RGP for $\phi$ with respect to the reduced game of (3.2).

Let $(N, v)$ be a game, $i \in N, j \in N-i, x=\phi(N, v)$, and let $\left(N-i, v^{x}\right)$ be the corresponding reduced game of (3.2). We must show that $\phi_{j}\left(N-i, v^{x}\right)=\phi_{j}(N, v)$.

Put $n:=|N|$. It follows from (2.3) that

$$
\phi_{l}(N, v)-\sum_{s \in \Gamma_{\mu l}} \gamma_{N}(S)\left[v\left(S^{c}\right)-v(S)\right]=\phi_{J}(N, v)-\sum_{s \in \Gamma_{\iota \jmath}} \gamma_{N}(S)\left[v\left(S^{c}\right)-v(S)\right] .
$$

By (2.3) and (3.2), we also have

$$
\begin{aligned}
\phi_{J}\left(N-i, v^{x}\right)= & \sum_{S \subset\{i, J\}^{c}} \gamma_{N-i}(S)\left[v^{x}((N-i)-S)-v^{x}(S)\right] \\
= & (n-1)^{-1} \sum_{S \subset\{i, J\}^{c}}|S| \gamma_{N-l}(S)\left[v\left((S \cup i)^{c}\right)-v(S \cup i)\right] \\
& +(n-1)^{-1} \sum_{S \subset\{l, J\}^{c}}(n-|S|-1) \gamma_{N-l}(S)\left[v\left(S^{c}\right)-v(S)\right] \\
& +(n-1)^{-1} x_{l} \sum_{S \subset\{i, J\}^{c}}(2|S|-n+1) \gamma_{N-i}(S) .
\end{aligned}
$$

Note that (3.8) is equivalent to

$$
\begin{aligned}
& (n-1)^{-1} x_{i} \sum_{s=0}^{n-2}\left(\begin{array}{c}
n-2 \\
s
\end{array}\right)(2 s-n+1)[(n-1) !]^{-1} s !(n-s-2) ! \\
& =(n-1)^{-2} x_{l} \sum_{s=0}^{n-2}(2 s-n+1)=-(n-1)^{-1} x_{i} .
\end{aligned}
$$

Furthermore, (3.7) is equivalent to

$$
n(n-1)^{-1} \sum_{S \subset\{l, J\}^{c}} \gamma_{N}(S)\left[v\left(S^{c}\right)-v(S)\right],
$$


while (3.6) is equivalent to

$$
\begin{aligned}
& (n-1)^{-1} \sum_{T \in \Gamma_{\imath \jmath}}(|T|-1) \gamma_{N-i}(T-i)\left[v\left(T^{c}\right)-v(T)\right] \\
& =n(n-1)^{-1} \sum_{T \in \Gamma_{l \jmath}} \gamma_{N}(T)\left[v\left(T^{c}\right)-v(T)\right] \\
& -(n-1)^{-1} \sum_{T \in \Gamma_{y}} \gamma_{N-i}(T-i)\left[v\left(T^{c}\right)-v(T)\right] \\
& =n(n-1)^{-1}\left[x_{\jmath}-\sum_{T \subset\{i, J\}^{c}} \gamma_{N}(T)\left[v\left(T^{c}\right)-v(T)\right]\right] \\
& -(n-1)^{-1} \sum_{T \in \Gamma_{l j}} \gamma_{N-i}(T-i)\left[v\left(T^{c}\right)-v(T)\right] \\
& =x_{J}+(n-1)^{-1} x_{l}+(n-1)^{-1} \sum_{T \in \Gamma_{l \jmath}}\left[\gamma_{N}(T)-\gamma_{N-i}(T-i)\right]\left[v\left(T^{c}\right)-v(T)\right] \\
& -(n-1)^{-1} \sum_{T \in \Gamma_{\mu \iota}} \gamma_{N}(T)\left[v\left(T^{c}\right)-v(T)\right] \\
& -n(n-1)^{-1} \sum_{T \subset\{i, j\}^{c}} \gamma_{N}(T)\left[v\left(T^{c}\right)-v(T)\right] \\
& =x_{J}+(n-1)^{-1} x_{i}-n(n-1)^{-1} \sum_{T \subset\{i, J\}^{c}} \gamma_{N}(T)\left[v\left(T^{c}\right)-v(T)\right],
\end{aligned}
$$

where the last equality follows from $\gamma_{N}(T)-\gamma_{N-\imath}(T-i)=-\gamma_{N}\left(T^{c}\right)$ for any $T \in \Gamma_{\iota}$ and by noting that $T \in \Gamma_{l \jmath}$ if and only if $T^{c} \in \Gamma_{\jmath l}$.

Thus, the sum of (3.6)-(3.8) is equal to the sum of (3.9)-(3.11), which equals $x_{j}$. Hence, $\phi_{J}\left(N-i, v^{x}\right)=x_{J}=\phi_{J}(N, v)$, i.e., the Shapley value possesses the reduced game property with respect to the reduced game of (3.2).

The existence proof of Theorem 3.2 is also valid for Theorem 3.3. We need only consider that the equality of (3.5) to the sum of (3.6)-(3.8) holds for the reduced game of (3.3) instead of the reduced game of (3.2).

General setting of the uniqueness proof of Theorems 3.1, 3.2, and 4.2. Let $\sigma$ and $\psi$ be two values on $G$ that possess the following properties: RISE, SYM (or ETP, respectively), and RGP with respect to a specified type of reduced games. We prove by induction on $|N|$ that $\sigma(N, v)=\psi(N, v)$ for any game $(N, v)$. The case $|N|=1$ is trivial because of Pareto-optimality, while the case where $|N|=2$ follows from Lemma 2.1. Thus, let $(N, v) \in G$ with $|N| \geqq 3$ and suppose that

$$
\sigma(M, w)=\psi(M, w) \quad \text { for all }(M, w) \in G \quad \text { with } 1 \leqq|M|<|N| .
$$

Define $(N, u) \in G$ by

$$
u(S):=v(S)-\sum_{J \in S} \sigma_{J}(N, v) \quad \text { for all } S \subset N .
$$

Since both values $\sigma$ and $\psi$ possess RISE, we have

$$
\sigma_{J}(N, u)=0 \quad \text { for all } j \in N \quad \text { and } \quad \psi(N, u)=\psi(N, v)-\sigma(N, v) .
$$

To show $\sigma(N, v)=\psi(N, v)$, it is equivalent to show that

$$
\psi_{j}(N, u)=0 \quad \text { for all } j \in N .
$$


Let $x=\sigma(N, v), i \in N$, and $\left(N-i, v^{x}\right)$ be the corresponding reduced game (according to the specified type of reduced games).

Define $(N-i, w) \in G$ by

$$
w(S):=v^{x}(S)-\sum_{J \in S} \sigma_{J}(N, v) \quad \text { for all } S \subset N-i .
$$

Let $y=\sigma(N, u)$. Then $y_{j}=0$ for all $j \in N$. Now we assert that the two operations of game reduction and zero-normalization with respect to the value $\sigma$ give rise to a commutative diagram. To be exact, the first assertion is that

$$
(N-i, w)=\left(N-i, u^{y}\right) .
$$

Then we deduce for all $j \in N-i$

$$
\begin{aligned}
\psi_{J}\left(N-i, u^{y}\right) & =\psi_{j}(N-i, w)=\psi_{J}\left(N-i, v^{x}\right)-\sigma_{J}(N, v) \\
& =\sigma_{J}\left(N-i, v^{x}\right)-\sigma_{J}(N, v)=\sigma_{J}(N, v)-\sigma_{J}(N, v)=0,
\end{aligned}
$$

where the equalities follow from (3.13), RISE of $\psi$, the induction hypothesis, and RGP of $\sigma$, respectively.

The second assertion is that $\psi_{j}\left(N-i, u^{y}\right)=0$ for all $i \in N$ and for all $j \in N-i$ implies (3.12). This completes the inductive proof of uniqueness.

Proof of the uniqueness part of Theorem 3.1. Consider the above general setting where $\psi:=\phi$ and the reduced games $\left(N-i, v^{x}\right):=\left(N-i, v^{\sigma}\right)$ and $\left(N-i, u^{y}\right):=$ $\left(N-i, u^{\sigma}\right)$ are given by (3.1). First, we prove (3.13).

Indeed, for any $S \subset N-i$ we have by RISE of $\sigma$ that $\sigma_{i}(S \cup i, u)=\sigma_{l}(S \cup i, v)-$ $\sigma_{l}(N, v)$ and hence,

$$
\begin{aligned}
u^{\sigma}(S) & =u(S \cup i)-\sigma_{i}(S \cup i, u)=v(S \cup i)-\sum_{\jmath \in S \cup i} \sigma_{\jmath}(N, v)-\sigma_{i}(S \cup i, v)+\sigma_{i}(N, v) \\
& =v(S \cup i)-\sum_{\jmath \in S} \sigma_{J}(N, v)-\sigma_{i}(S \cup i, v)=v^{\sigma}(S)-\sum_{\jmath \in S} \sigma_{J}(N, v)=w(S) .
\end{aligned}
$$

Thus, (3.13) holds.

Finally, we prove the second assertion in the general setting. By (3.1) and the induction hypothesis, we have that $u^{\sigma}(S)=u^{\phi}(S)$ for all $S \subset N-i, S \neq N-i$, while $u^{\sigma}(N-i)=u^{\phi}(N-i)+\phi_{i}(N, u)$ since $\sigma_{l}(N, u)=0$. By $(2.1)$, this implies that for all $j \in N-i$

$$
\phi_{J}\left(N-i, u^{\sigma}\right)=\phi_{J}\left(N-i, u^{\phi}\right)+(|N|-1)^{-1} \phi_{i}(N, u)
$$

and hence,

$$
\begin{aligned}
0 & =\phi_{J}\left(N-i, u^{\sigma}\right)=\phi_{J}\left(N-i, u^{\phi}\right)+(|N|-1)^{-1} \phi_{l}(N, u) \\
& =\phi_{J}(N, u)+(|N|-1)^{-1} \phi_{i}(N, u)
\end{aligned}
$$

by using the fact that the Shapley value $\phi$ possesses RGP. Therefore, $\phi_{J}(N, u)=$ $-(|N|-1)^{-1} \phi_{l}(N, u)$ for all $j \in N-i$. From this and $|N| \geqq 3$, we conclude that $\phi_{J}(N, u)=\phi_{k}(N, u)$ for all $j, k \in N$. Now it follows from the Pareto-optimality and $u(N)=0$ that $\phi_{J}(N, u)=0$ for all $j \in N$. Thus, (3.12) holds. This completes the proof of uniqueness of Theorem 3.1.

Proof of the uniqueness part of Theorem 3.2. Consider the above general setting where $\psi:=\phi$ and the reduced games are given by (3.2). Put $n:=|N|$. First, we prove (3.13). 
For any $S \subset N-i$ we have

$$
\begin{aligned}
u^{y}(S) & =(n-1)^{-1}|S|\left[u(S \cup i)-y_{i}\right]+(n-1)^{-1}(n-|S|-1) u(S) \\
& =(n-1)^{-1}|S|[v(S \cup i)-x(S \cup i)]+(n-1)^{-1}(n-|S|-1)[v(S)-x(S)] \\
& =(n-1)^{-1}|S|\left[v(S \cup i)-x_{i}\right]+(n-1)^{-1}(n-|S|-1) v(S)-x(S) \\
& =v^{x}(S)-x(S)=w(S) .
\end{aligned}
$$

Thus, (3.13) holds.

Finally, we prove the second assertion in the general setting. By using the alternative formula (2.3), we obtain that for all $j \in N-i$

$$
\begin{aligned}
0= & \phi_{j}\left(N-i, u^{y}\right)=\sum_{S \subset\{l, J\}^{c}} \gamma_{N-i}(S)\left[u^{y}((N-i)-S)-u^{y}(S)\right] \\
= & (n-1)^{-1} \sum_{S \subset\{l, J\}^{c}} \gamma_{N-i}(S)\left[(n-|S|-1)\left[u\left(S^{c}\right)-u(S)\right]\right. \\
& \left.\quad+|S|\left[u\left((S \cup i)^{c}\right)-u(S \cup i)\right]\right] \\
= & n(n-1)^{-1} \sum_{S \subset\{i, j\}^{c}} \gamma_{N}(S)\left[u\left(S^{c}\right)-u(S)\right] \\
& +(n-1)^{-1} \sum_{T \in \Gamma_{\nu j}}(|T|-1) \gamma_{N-i}(T-i)\left[u\left(T^{c}\right)-u(T)\right] \\
= & n(n-1)^{-1} \phi_{J}(N, u)-(n-1)^{-1} \sum_{T \in \Gamma_{l j}} \gamma_{N-i}(T-i)\left[u\left(T^{c}\right)-u(T)\right] .
\end{aligned}
$$

Thus,

$$
\phi_{j}(N, u)=n^{-1} \sum_{T \in \Gamma_{\imath \jmath}} \gamma_{N-l}(T-i)\left[u\left(T^{c}\right)-u(T)\right] \quad \text { for all } i, j \in N, \quad i \neq j .
$$

By interchanging the players $i$ and $j$, we obtain that for all $i, j \in N, i \neq j$,

$$
\begin{aligned}
\phi_{i}(N, u) & =n^{-1} \sum_{T \in \Gamma_{j l}} \gamma_{N-\jmath}(T-j)\left[u\left(T^{c}\right)-u(T)\right] \\
& =n^{-1} \sum_{S \in \Gamma_{\imath \jmath}} \gamma_{N-i}(S-i)\left[u(S)-u\left(S^{c}\right)\right]=-\phi_{J}(N, u) .
\end{aligned}
$$

From this and $|N| \geqq 3$, we conclude that for any three players $i, j, k \in N$ we have on the one hand, $\phi_{i}(N, u)=-\phi_{j}(N, u)$, and on the other hand, $\phi_{i}(N, u)=-\phi_{k}(N, u)=$ $\phi_{J}(N, u)$. It follows that $\phi_{J}(N, u)=0$ for all $j \in N$. Thus, (3.12) holds. This completes the proof of uniqueness of Theorem 3.2.

It is still an open problem to (dis)prove the conjecture that the Shapley value on $G$ is the unique value on $G$ which possesses RISE, SYM, and RGP with respect to the reduced game of (3.3). For the sake of completeness, we remark that the general setting of the uniqueness proofs does not apply here since (3.13) fails to hold.

4. On the functional equations determining the prekernel and the prenucleolus. Two well-known solutions are the kernel, introduced in Davis and Maschler [6], and the nucleolus, introduced in Schmeidler [23] as a one-point solution within the kernel. Closely related to the kernel is the prekernel, which was introduced in Maschler, Peleg, and Shapley [17]. The prekernel can be seen as a simplification of the kernel, while the prenucleolus is defined in the same way as the nucleolus, except it omits IR. In this 
section we review the axiomatizations of both the prekernel and the prenucleolus by means of a consistency property. First, we recall the definition of the prekernel.

The prekernel is based on the ideas of excess and maximum surplus. The excess of coalition $S$ in a game $(N, v)$ with respect to a payoff vector $x \in \mathbb{R}^{N}$ is defined to be

$$
e(S, x, N, v):=v(S)-x(S),
$$

where $e(\varnothing, x, N, v):=0$. The excess of $S$ at $x$ represents the gain (or loss, if it is negative) to the coalition $S$ in the game if its members withdraw from the payoff vector $x$ in order to form their own coalition. The maximum surplus of a player $i \in N$ over another player $j \in N$ in a game $(N, v)$ with respect to a payoff vector $x \in \mathbb{R}^{N}$ is given by

$$
s_{i j}(x, N, v):=\max [v(S)-x(S) \mid S \subset N, i \in S, j \notin S] .
$$

The maximum surplus of $i$ over $j$ at $x$ represents the maximal amount that player $i$ can gain (or the minimal amount that $i$ can lose, if it is negative) without the cooperation of player $j$ by withdrawing from the payoff vector $x$ and forming a coalition not containing $j$, on the understanding that the other members of the coalition are satisfied with the amount they had according to $x$. Thus, the maximum surplus $s_{l j}(x, N, v)$ can be seen as a measure of the power of $i$ to threaten $j$ in the game $(N, v)$ with respect to the payoff vector $x$. The prekernel consists of pre-imputations for which no player is more powerful than another player. That is, the prekernel of a game $(N, v)$ is defined to be

$$
\mathscr{K}^{*}(N, v):=\left\{x \in I^{*}(N, v) \mid s_{i j}(x, N, v)=s_{j l}(x, N, v) \text { for all } i, j \in N, i \neq j\right\} .
$$

The following type of reduced games is due to Davis and Maschler [6] and the corresponding RGP proved to be of importance for the development of the theory of the kernel (see [16], [17]). The reduced game in question is derived from the original game by removing several players, on the understanding that the removed players are paid according to the payoff vector $x$. The total earnings in the reduced game are equal to the total earnings in the original game minus the total payoff to the removed players. Furthermore, the worth of a proper coalition in the reduced game is obtained as the most profitable of several possibilities to cooperate with removed players, taking into account that those players are paid according to $x$. Note that the relevant reduced game differs from the foregoing reduced games by the fact that the maximum overall subsets of the set of the removed players appears for the first time in the definition of a reduced game.

Given any game $(N, v)$, any coalition $T \subset N$, and any payoff vector $x \in \mathbb{R}^{N}$, the corresponding reduced game $\left(T, v^{x}\right)$ with respect to $x$ is as follows:

$$
\begin{aligned}
& v^{x}(\varnothing):=0, \quad v^{x}(T):=v(N)-x(N-T), \quad \text { for all other } S \subset T, \\
& v^{x}(S):=\max [v(S \cup R)-x(R) \mid R \subset N-T] .
\end{aligned}
$$

In case the payoff vector $x$ is Pareto-optimal, the reduced game of (4.3) agrees with the reduced game as defined in Davis and Maschler [6]. It is known and straightforward to verify that for any game $(N, v)$, any two players $i, j \in N, i \neq j$, and any payoff vector $x \in \mathbb{R}^{N}$

$$
s_{i j}(x, N, v)=s_{\jmath l}(x, N, v)+x_{J}-x_{\iota}
$$

if $i$ and $j$ are substitutes in the game $(N, v)$;

$$
s_{i j}\left(x^{T}, T, v^{x}\right)=s_{i j}(x, N, v) \text { for any } T \subset N \text { with }\{i, j\} \subset T,
$$

where the reduced game $\left(T, v^{x}\right)$ is given by $(4.3)$. 
By (4.4), it is obvious that the prekernel possesses ETP. Furthermore, it follows immediately from (4.5) that the prekernel possesses RGP with respect to the reduced game of (4.3). Closely related to RGP is the converse reduced game property, which refers to two-person reduced games. A solution $\sigma$ is said to possess the converse reduced game property (CRGP) (with respect to a specified type of reduced games) whenever the next condition is satisfied.

CRGP. If $(N, v)$ is a game and $x \in I^{*}(N, v)$ such that $x^{T} \in \sigma\left(T, v^{x}\right)$ for all $T \subset N$ with $|T|=2$, then $x \in \sigma(N, v)$. The converse reduced game property requires that a pre-imputation $x \in \mathbb{R}^{N}$ is in the solution set $\sigma(N, v)$ of the original game $(N, v)$ whenever its restriction $x^{T}$ to any pair $T$ of players is in the solution set of the corresponding reduced game $\left(T, v^{x}\right)$. We remark that CRGP was already used by Harsanyi [12] in his study on bargaining games. It is a direct consequence of (4.5) that the prekernel also possesses CRGP with respect to the reduced game of (4.3). Clearly, the prekernel has RISE, while the nonemptiness of the prekernel was already established in Maschler, Peleg, and Shapley [17]. Peleg [20] proved that these five properties fully characterize the prekernel.

THEOREM 4.1 (Peleg [20]). The prekernel $\mathscr{K}^{*}$ on $G$ is the unique solution on $G$ that possesses NE, RISE, ETP, RGP, and CRGP with respect to the reduced game of (4.3).

Proof. It remains to prove uniqueness. Let $\sigma$ be a solution on $G$ which possesses the following five properties: NE, RISE, ETP, RGP, and CRGP. We show by induction on $|N|$ that $\sigma(N, v)=\mathscr{K}^{*}(N, v)$ for any game $(N, v)$. The case $|N|=1$ is trivial and the case $|N|=2$ follows from Lemma 2.1. So, let $(N, v) \in G$ with $|N| \geqq 3$ and suppose that $\sigma(M, w)=\mathscr{K}^{*}(M, w)$ for all $(M, w) \in G$ with $1 \leqq|M|<|N|$. Let $x \in \sigma(N, v)$. Then for any $T \subset N$ with $|T|=2$ we have $x^{T} \in \sigma\left(T, v^{x}\right)=\mathscr{K}^{*}\left(T, v^{x}\right)$ by RGP of $\sigma$ and the induction hypothesis. Now we conclude from CRGP of $\mathscr{K}^{*}$ that $x \in$ $\mathscr{K}^{*}(N, v)$. Hence, $\sigma(N, v) \subset \mathscr{K}^{*}(N, v)$, while the inverse inclusion can be obtained by interchanging the solutions $\sigma$ and $\mathscr{K}^{*}$. Thus, $\sigma(N, v)=\mathscr{K}^{*}(N, v)$. This completes the inductive proof of uniqueness.

Peleg's proof of Theorem 4.1 is remarkably short and elegant in contrast to Sobolev's proof of his axiomatization of the prenucleolus by means of RGP with respect to the reduced game of (4.3). The prenucleolus is defined as follows.

Let $(N, v)$ be a game and write $n=|N|$. For any payoff vector $x \in \mathbb{R}^{N}$, let $\theta(x)$ be the $2^{n}$-tuple whose components are the excesses $e(S, x, N, v), S \subset N$, arranged in nonincreasing order. That is, we have

$$
\theta_{l}(x) \geqq \theta_{J}(x) \quad \text { whenever } 1 \leqq i \leqq j \leqq 2^{n} .
$$

Now we order the various "complaint vectors" $\theta(x), x \in \mathbb{R}^{N}$, by the lexicographic order $\leqq_{L}$ on $\mathbb{R}^{2^{n}}$. For $x, y \in \mathbb{R}^{N}$ we write

$$
\begin{array}{ll}
\theta(x)<_{L} \theta(y) \quad & \text { if there exists an integer } 1 \leqq k \leqq 2^{n} \text { such that } \\
& \theta_{l}(x)=\theta_{l}(y) \text { for } 1 \leqq i<k \text { and } \theta_{k}(x)<\theta_{k}(y) ; \\
\theta(x) \leqq_{L} \theta(y) \quad \text { if either } \theta(x)=\theta(y) \text { or } \theta(x){ }_{L} \theta(y) .
\end{array}
$$

The prenucleolus consists of pre-imputations which minimize the complaint function $\theta(x)$ in the lexicographic order over the nonempty convex pre-imputation set. That is, the prenucleolus of a game $(N, v)$ is defined to be

$$
\mathscr{N}^{*}(N, v):=\left\{x \in I^{*}(N, v) \mid \theta(x) \leqq_{L} \theta(y) \text { for all } y \in I^{*}(N, v)\right\} .
$$

It is well known that the prenucleolus of any game $(N, v)$ consists of a unique point which is usually denoted by $n^{*}(N, v)$. Obviously, the prenucleolus has SYM and RISE. 
Furthermore, in order to characterize the prenucleolus by means of a consistency property, it was Sobolev [28] who introduced RGP with respect to the reduced game of (4.3).

THEOREM 4.2 (Sobolev [28]). The prenucleolus $n^{*}$ on $G$ is the unique value on $G$ that possesses RISE, SYM, and RGP with respect to the reduced game of (4.3).

For the proof of the involved consistency property for the prenucleolus and of the uniqueness part of the above theorem, we refer to Sobolev [28]. However, it is also possible to put the proof of the uniqueness part of Theorem 4.2 in the general setting of the uniqueness proofs as treated in $\S 3$. The first assertion (3.13) in the general setting can easily be shown (see below), but unfortunately the second and last assertion in the general setting is still open to be proved. This open problem concerning the prenucleolus, which is interesting in itself, is stated below the proof of the assertion (3.13).

Proof of (3.13). Consider the general setting of the uniqueness proofs as treated in $\S 3$, where $\psi:=n^{*}$ and the reduced games $\left(N-i, v^{x}\right)$ and $\left(N-i, u^{y}\right)$ are given by (4.3). Since $y_{J}=0$ for all $j \in N$, we have

$$
u^{y}(N-i)=u(N)-y_{i}=u(N)=v(N)-x(N)=0,
$$

while for any other coalition $S \subset N-i$

$$
\begin{aligned}
u^{y}(S) & =\max \left[u(S \cup i)-y_{l}, u(S)\right]=\max [u(S \cup i), u(S)] \\
& =\max [v(S \cup i)-x(S \cup i), v(S)-x(S)]=\max \left[v(S \cup i)-x_{l}, v(S)\right]-x(S) \\
& =v^{x}(S)-x(S)=w(S) .
\end{aligned}
$$

Because $w(N-i)=v^{x}(N-i)-x(N-i)=v(N)-x_{i}-x(N-i)=v(N)-x(N)=$ 0 , it follows that $w(S)=u^{y}(S)$ for all $S \subset N-i$. Thus, (3.13) holds.

OPEN PROBLEM 4.3. Let $(N, u)$ be a game with $|N| \geqq 3$ and $u(N)=0$.

For any $i \in N$, let the game $\left(N-\{i\}, w_{i}\right)$ be defined by

$$
w_{i}(\varnothing):=0, \quad w_{l}(N-\{i\}):=0 \quad \text { and } \quad w_{i}(S):=\max [u(S \cup\{i\}), u(S)]
$$

for all other $S \subset N-\{i\}$. If $n_{j}^{*}\left(N-\{i\}, w_{i}\right)=0$ for all $i \in N$ and all $j \in N-\{i\}$, then $n_{j}^{*}(N, u)=0$ for all $j \in N$.

Note the resemblance of the open problem to the converse reduced game property. In fact, the open problem states that the prenucleolus possesses CRGP, which refers to the $(|N|-1)$-person (instead of the two-person) reduced games of (4.3).

5. On the functional equations determining the core. In view of the definition given in $\S 1$, the core of a game may be empty. A game $(N, v)$ is called balanced if its core $C(N, v) \neq \varnothing$, and it is called totally balanced if the core $C(T, v)$ of any subgame $(T, v)$ is nonempty. The classes of balanced and totally balanced games have been studied in many papers. A balanced game can be characterized in terms of balanced collections of coalitions ( see [4] and [25]), while the class of totally balanced games is characterized as the class of market games [26] and of flow games [14]. In this section we review the axiomatic characterization of the core of balanced games as well as of the core of totally balanced games, due to Peleg [20], [21].

First, we list the superadditivity property, which requires that the algebraic sum of the solution sets of two games with the same player set is contained in the solution set of the sum of the two involved games.

SAP. A solution $\sigma$ is said to possess the superadditivity property (SAP) if

$$
\sigma(N, v+w) \supset \sigma(N, v)+\sigma(N, w) \text { for all games }(N, v) \text { and }(N, w) .
$$


Note that for values, SAP is equivalent to the additivity property mentioned at the beginning of $\S 3$. Clearly, the core is both superadditive and individually rational. It is also straightforward to verify that the core on $G$ possesses RGP with respect to the reduced game of (4.3). It turns out that these three properties together with NE fully characterize the core of balanced games. For the uniqueness proof we refer to Peleg [20].

THEOREM 5.1 (Peleg [20]). The core C is the unique solution on the class of balanced games which possesses NE, IR, SAP, and RGP with respect to the reduced game of (4.3).

The reduced game of (4.3) corresponding to a (totally) balanced game is itself also balanced, but it may fail to be totally balanced. Hence, the restriction of the core to the class of totally balanced games does not possess RGP on this class. However, for games with at most two players total balancedness is equivalent to balancedness. Therefore, the core on the class of totally balanced games still possesses the weak reduced game property, which refers to one- or two-person reduced games. A solution $\sigma$ on a class $\mathscr{G}$ of games is said to possess the weak reduced game property (WRGP) (with respect to a specified type of reduced games) whenever the next condition is satisfied:

WRGP. If $(N, v) \in \mathscr{G}, T \subset N, 1 \leqq|T| \leqq 2$ and $x \in \sigma(N, v)$, then $\left(T, v^{x}\right) \in \mathscr{G}$ and $x^{T} \in \sigma\left(T, v^{x}\right)$.

The following theorem provides an axiomatic characterization of the core of totally balanced games by means of WRGP and CRGP.

THEOREM 5.2 (Peleg [21]). The core $C$ is the unique solution on the class of totally balanced games which possesses NE, IR, SAP, WRGP, CRGP with respect to the reduced game of (4.3) (where all properties are restricted to the class of totally balanced games).

Proof. We already noted that the core on the class of totally balanced games possesses NE, IR, SAP, and WRGP. Finally, we establish that the core on $G$ possesses CRGP.

Let $(N, v)$ be any game and $x \in I^{*}(N, v)$ such that $x^{T} \in C\left(T, v^{x}\right)$ for any $T \subset N$ with $|T|=2$. We must show that $x(S) \geqq v(S)$ for $S \subset N, S \neq \varnothing, N$. Choose $i \in S, j \in$ $N-S$, and let $T=\{i, j\}$. Now $x^{T} \in C\left(T, v^{x}\right)$ implies $x_{l} \geqq v^{x}(\{i\})$, while the definition of $v^{x}(\{i\})$ in the reduced game $\left(T, v^{x}\right)$ yields $v^{x}(\{i\}) \geqq v(S)-x(S-\{i\})$. It follows that $x_{l} \geqq v(S)-x(S-\{i\})$ and hence, $x(S) \geqq v(S)$. So, $x \in C(N, v)$ and CRGP holds for the core on $G$. For the proof of uniqueness we refer to [21]. The uniqueness proof of the solution for games with at least three players resembles the uniqueness proof of Theorem 4.1.

Peleg [21] also proved that in Theorem 5.2, NE and SAP can be replaced by the strong requirement for a solution $\sigma$ that for any balanced two-person game $(N, v)$

$$
\sigma(N, v)=\left\{x \in \mathbb{R}^{N} \mid \sum_{j \in N} x_{j}=v(N) \text { and } x_{i} \geqq v(\{i\}) \text { for all } i \in N\right\} .
$$

This requirement for the solution $\sigma$ simply states that the solution coincides with the core for two-person games. In [18], a geometric characterization of the intersection of the prekernel with the core is presented. In view of Theorems 4.1 and 5.2, the intersection of the prekernel with the core satisfies RISE, IR, ETP, WRGP (of totally balanced games), and CRGP with respect to the reduced game of (4.3). Together with NE, these five properties fully characterize the intersection of the prekernel with the core on the class of totally balanced games ( see [21]).

6. An example and an application. The final section is devoted to an example that illustrates the theory of $\S \S 3$ and 4 , and also to an application of the reduced game property to the bankruptcy problem as presented in Aumann and Maschler [2]. 
6.1. Example. Let the three-person game $(N, v)$ be defined by $N=\{1,2,3\}$ and $v(\{i\})=0$ for all $i \in N, v(\{1,2\})=90, v(\{1,3\})=v(\{2,3\})=-30$, and $v(N)=$ 60 . The above game is strategically equivalent to the game corresponding to [18, Fig. 5, p. 318].

Let $x=\left(x_{1}, x_{2}, x_{3}\right) \in \mathbb{R}^{N}$ and $\sigma$ any value which is standard for two-person games. Thus, $\sigma_{t}(\{i, j\}, v)=\frac{1}{2} v(\{i, j\})$ for all $i, j \in N, i \neq j$.

The corresponding two-person reduced games of (3.1)-(3.3) and (4.3), respectively, are listed in columns $2-4,5-7,8-10$, and 11-13 of Table 1 , while the payoff vectors for the corresponding two-person reduced games according to the value $\sigma$ are listed in rows 9-11. For notational reasons, we write $\alpha_{+}$instead of $\max [0, \alpha]$, where $\alpha \in \mathbb{R}$. Thus, $\alpha_{+}$ $=\alpha$ if $\alpha \geqq 0$ and $\alpha_{+}=0$ if $\alpha \leqq 0$.

The Shapley value $\phi(N, v)$ of the original game $(N, v)$ can now be determined by solving the functional equations in terms of the reduced games of (3.1), (3.2), or (3.3). That is, by solving the following system of linear equations:

$$
\begin{aligned}
& x_{1}=30-\frac{1}{2} x_{3}, \quad x_{1}=60-\frac{1}{2} x_{2}, \quad x_{2}=60-\frac{1}{2} x_{1}, \\
& x_{2}=30-\frac{1}{2} x_{3}, \quad x_{3}=-\frac{1}{2} x_{2}, \quad x_{3}=-\frac{1}{2} x_{1} .
\end{aligned}
$$

The unique solution of this system is $x=(40,40,-20)$. Hence, the Shapley value $\phi(N, v)=(40,40,-20)$; of course, this payoff vector can also be calculated by applying formula (2.1) or (2.3).

The prenucleolus $n *(N, v)$ can be obtained by solving the functional equations in terms of the reduced games of (4.3), i.e., by solving the following system of equations:
(1) $x_{1}=30-\frac{1}{2} x_{3}$,
$x_{1}=30-\frac{1}{2} x_{2}+\frac{1}{2}\left(90-x_{2}\right)_{+}-\frac{1}{2}\left(-30-x_{2}\right)_{+}$,
(2) $x_{2}=30-\frac{1}{2} x_{3}$
(4) $x_{3}=30-\frac{1}{2} x_{2}+\frac{1}{2}\left(-30-x_{2}\right)_{+}-\frac{1}{2}\left(90-x_{2}\right)_{+}$,

and

$$
\begin{aligned}
& x_{2}=30-\frac{1}{2} x_{1}+\frac{1}{2}\left(90-x_{1}\right)_{+}-\frac{1}{2}\left(-30-x_{1}\right)_{+} \\
& x_{3}=30-\frac{1}{2} x_{1}+\frac{1}{2}\left(-30-x_{1}\right)_{+}-\frac{1}{2}\left(90-x_{1}\right)_{+}
\end{aligned}
$$

Since $x_{1}=x_{2}$ by (1) and (2), it follows from (3) that

$$
3 x_{2}-60=\left(90-x_{2}\right)_{+}-\left(-30-x_{2}\right)_{+} .
$$

Hence, $x_{2}=75 / 2$ and the unique solution of the above system is $x=(75 / 2,75 / 2,-15)$. Thus, the prenucleolus $n^{*}(N, v)=(75 / 2,75 / 2,-15)$. Since $\mathscr{K}^{*}=n^{*}$ for two-person games, we have by RGP of $\mathscr{K}^{*}$ that $\mathscr{K}^{*}(N, v)=\left\{n^{*}(N, v)\right\}$.

Because $n^{*}(N, v) \neq \phi(N, v)$, it follows that the Shapley value (prenucleolus, respectively) does not possess RGP with respect to the reduced game of (4.3) ((3.1), (3.2), or (3.3)). Note that both the Shapley value and the prenucleolus are not individually rational. For the sake of completeness, we recall that the kernel $\mathscr{K}(N, v)=$ $\{n(N, v)\}=\{(30,30,0)\}($ see $[18$, p. 317]).

6.2. Application. A bankruptcy problem is defined as a pair $\left(E ; d_{1}, d_{2}, \cdots, d_{n}\right)$, where $E$ represents the estate of a man who died, leaving debts $d_{1}, d_{2}, \cdots, d_{n}$ to $n$ creditors. It is supposed that the debts are arranged in nondecreasing order (i.e., $0 \leqq$ $d_{1} \leqq d_{2} \leqq \cdots \leqq d_{n}$ ) and that the total debt is at least the estate (i.e., $0 \leqq E \leqq d_{1}+$ 


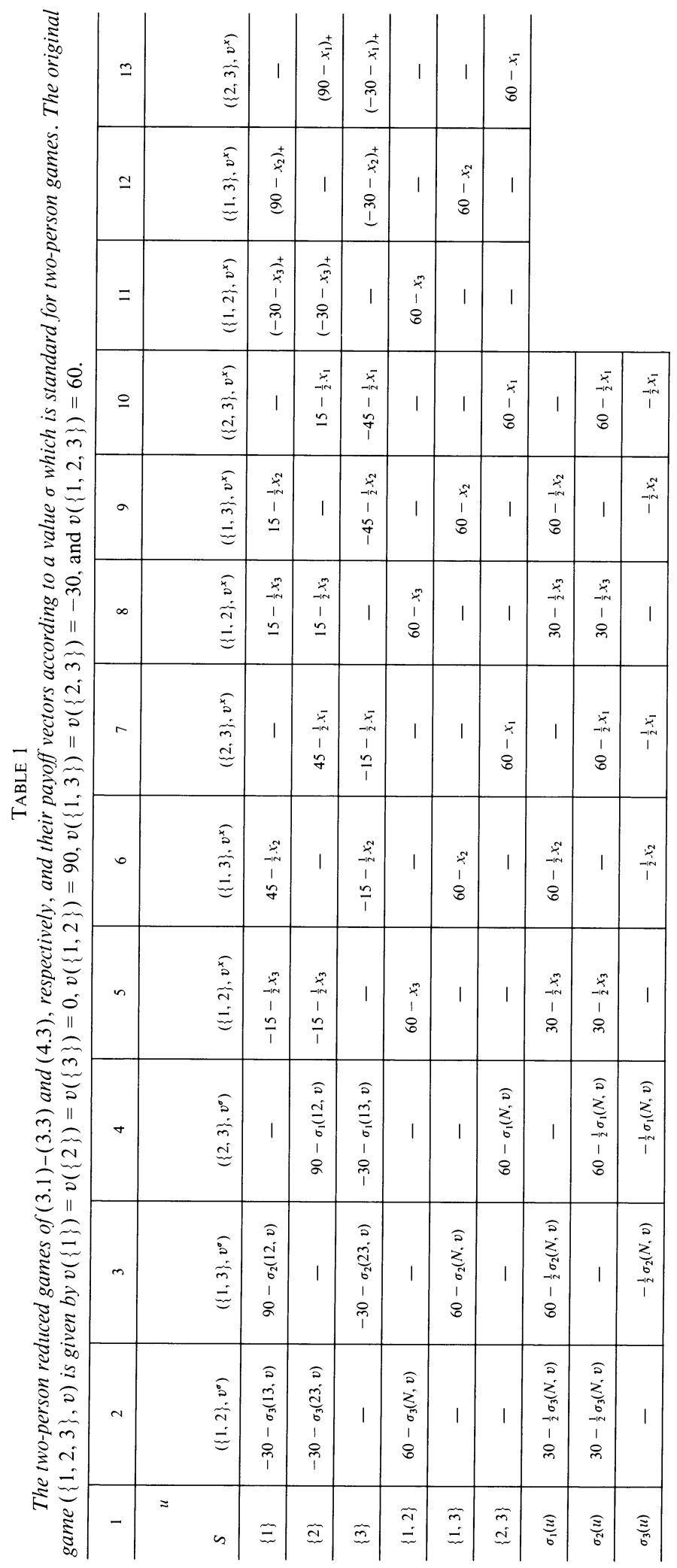


TABLE 2

The relationship between solutions and properties. Here $+(-$, respectively $)$ denotes the (in) validity of the property for the solution. Recall that in general the prenucleolus is a proper subset of the prekernel.

\begin{tabular}{c|ccc}
\hline & & & \\
Solution & Prenucleolus \\
$n^{*}$ & $\begin{array}{c}\text { Prekernel } \\
\mathscr{K}^{*}\end{array}$ \\
\hline RGP & $(3.1),(3.2)$, or $(3.3)$ & $(4.3)$ & $(4.3)$ \\
\hline NE & + & + & + \\
\hline RISE & + & + & + \\
\hline ETP & + & + & + \\
\hline SYM & + & - & + \\
\hline CRGP $(4.3)$ & - & - & + \\
\hline IR & - & + & + \\
\hline
\end{tabular}

$\left.d_{2}+\cdots+d_{n}\right)$. The bankruptcy game $(N, v)$ corresponding to the bankruptcy problem $\left(E ; d_{1}, d_{2}, \cdots, d_{n}\right)$ is then defined as follows:

$$
\begin{aligned}
& N:=\{1,2, \cdots, n\} \quad \text { as the set of } n \text { creditors, } \\
& v(S):=\max \left[0, E-\sum_{J \in N-S} d_{j}\right] \quad \text { for all } S \subset N
\end{aligned}
$$

as the amount which the coalition $S$ can get without going to court, i.e., the nonnegative amount which is left of the estate $E$ after each member $j$ of the complement $N-S$ is paid his complete debt $d_{J}$. With the aid of RGP of the prekernel with respect to the reduced game of (4.3), it is established by Aumann and Maschler [2] that the nucleolus of the corresponding bankruptcy game is the unique "consistent" solution of the bankruptcy problem.

Finally, we remark that an extensive game theoretic analysis of the bankruptcy problem is presented in [9, Chap. VI]. The analysis elucidates the relationships between specific division rules for the bankruptcy problem and the game theoretic notions of the Shapley value, the nucleolus and the so-called $\tau$-value. Moreover, axiomatic characterizations of the $\tau$-value for the bankruptcy problem are treated in [5] and [9].

A consistency property for division rules in the context of the taxation problem has been considered in [32]. Further literature dealing with consistency, reduced games, and axiomatizations of solutions can be found in [8], [15], [19], and [29].

In summary, we list the results of Theorems 3.1-3.3 and 4.1, 4.2 in Table 2.

\section{REFERENCES}

[1] R. J. Aumann AND J. H. DRÈze, Cooperative games with coalition structures, Internat. J. Game Theory, 3 (1974), pp. 217-237. 
[2] R. J. AUMANN AND M. MASCHLER, Game theoretic analysis of a bankruptcy problem from the Talmud, J. Econom. Theory, 36 (1985), pp. 195-213.

[3] R. J. Aumann and L. S. Shapley, Values of Non-atomic Games, Princeton University Press, Princeton, NJ, 1974.

[4] O. N. BondAREVA, Some applications of linear programming methods to the theory of cooperative games, Problemy Kibernet., 10 (1963), pp. 119-139. (In Russian.)

[5] I. J. Curiel, M. Maschler, AND S. H. TiJs, Bankruptcy games, Z. Oper. Res. Ser. A, 31 (1987), pp. 143-159.

[6] M. Davis AND M. MASChler, The kernel of a cooperative game, Naval Res. Logist. Quart., 12 (1965), pp. 223-259.

[7] T. S. H. DriesSEN, A new axiomatic characterization of the Shapley value, Methods Oper. Res., 50 (1985), pp. 505-517.

[8] — - A relationship between reduced games and largeness of the core of a game, Methods Oper. Res., 54 (1986), pp. 349-361.

[9] - Cooperative Games, Solutions and Applications, Kluwer Academic Publishers, Dordrecht, the Netherlands, 1988.

[10] P. Dubey, On the uniqueness of the Shapley value, Internat. J. Game Theory, 4 (1975), pp. 131-139.

[11] D. B. Gillies, Some theorems on n-person games, Ph.D. thesis, Princeton University Press, Princeton, NJ, 1953.

[12] J. HARSANYI, A bargaining model for the cooperative n-person game, in Contributions to the Theory of Games IV, A. W. Tucker and R. D. Luce, eds., Ann. Math. Stud., 40, Princeton University Press, Princeton, NJ, 1959, pp. 325-355.

[13] S. HaRt ANd A. Mas-Colell, Potential, value and consistency, Econometrica, 57 ( 1989), pp. $589-614$.

[14] E. Kalai And E. Zemel, Totally balanced games and games of flow, Math. Oper. Res., 7 (1982), pp. 476-478.

[15] T. LENSBERG, Stability and collective rationality, Econometrica, 55 (1987), pp. 935-962.

[16] M. MASChler AND B. PELEG, The structure of the kernel of a cooperative game, SIAM J. Appl. Math., 15 (1967), pp. 569-604.

[17] M. Maschler, B. Peleg, AND L. S. Shapley, The kernel and bargaining set for convex games, Internat. J. Game Theory, 1 ( 1972), pp. 73-93.

[18] - Geometric properties of the kernel, nucleolus, and related solution concepts, Math. Oper. Res., 4 (1979), pp. 303-338.

[19] H. Moulin, Equal or proportional division of a surplus, and other methods, Internat. J. Game Theory, 16 (1987), pp. 161-186.

[20] B. PELEG, On the reduced game property and its converse, Internat. J. Game Theory, 15 (1986), pp. 187200; Correction, Internat. J. Game Theory, 16 (1987), p. 290.

[21] — An axiomatization of the core of market games, Math. Oper. Res., 14 (1989), pp. 448-456.

[22] J. S. RansmeIer, The Tennessee Valley Authority: A Case Study in the Economics of Multiple Purpose Stream Planning, Vanderbilt University Press, Nashville, 1942.

[23] D. SCHMEIDLER, The nucleolus of a characteristic function game, SIAM J. Appl. Math., 17 (1969), pp. 1163-1170.

[24] L. S. ShaPley, $A$ value for n-person games, in Contributions to the Theory of Games II, H. Kuhn and A. W. Tucker, eds., Ann. Math. Stud. 28, Princeton University Press, Princeton, NJ, 1953, pp. 307317.

[25] — On balanced sets and cores, Naval Res. Logist. Quart., 14 (1967), pp. 453-460.

[26] L. S. ShaPley AND M. SHubiK, On market games, J. Econom. Theory, 1 (1969), pp. 9-25.

[27] A. I. Sobolev, The functional equations that give the payoffs of the players in an nerson game, in Advances in Game Theory, E. Vilkas, ed., Izdat. "Mintis," Vilnius, 1973, pp. 151-153. (In Russian.)

[28] — The characterization of optimality principles in cooperative games by functional equations, in Mathematical Methods in the Social Sciences 6, N. N. Vorobev, ed., Vilnius, 1975, pp. 94-151. (In Russian.)

[29] W. Thomson And T. Lensberg, Axiomatic theory of bargaining with a variable number of agents, Cambridge University Press, Cambridge, 1989.

[30] J. VON NEUMANN AND O. MORGENSTERN, Theory of games and economic behavior, Princeton University Press, Princeton, NJ, 1944.

[31] H. P. YounG, Monotonic solutions of cooperative games, Internat. J. Game Theory, 14 ( 1985), pp. 6572.

[32] - On dividing an amount according to individual claims or liabilities, Math. Oper. Res., 12 (1987), pp. 398-414. 